\title{
Entrevista a Roberto Soto: Presidente de la Asociación de Profesionales de Bibliotecas Móviles (ACLEBIM)
}

\author{
FELICIDAD CAMPAL \\ Vocal de SEDIC
}

\begin{abstract}
Licenciado en Geografía e Historia por la Universidad de Valladolid, y en Documentación por la Univeritat Oberta de Catalunya.

Desde 1991 está ligado a los Bibliobuses de León, primero como encargado de bibliobús, y desde 2007 como Jefe de Sección de Coordinación de Bibliotecas en la Diputación de León.

Presidente de la Asociación de Profesionales de Bibliotecas Móviles (ACLEBIM).

Responsable de la Sección de Bibliobuses en la revista "Mi Biblioteca".

Colaborador en el blog colectivo Biblogtecarios.

Coordinador del Grupo de Trabajo de Especial Atención al Medio Rural del Ministerio de Cultura y Deporte.

Bajo su gestión, las entidades de las que se ocupa han conseguido distintos reconocimientos, como el Premio Nacional al Fomento de la Lectura (ACLEBIM), el Reconocimiento al mejor servicio digital en bibliotecas públicas de poblaciones inferiores a los 5.000 habitantes del Ministerio de Cultura (App “Bibliobuses de León”), o el Premio REBIUN al Plan de Modernización de los Bibliobuses de León.
\end{abstract}

Bibliobuses, servicios bibliotecarios, asociaciones bibliotecarias

Hace muchos años cuando aprobé la oposición mi destino fue la Biblioteca Pública de Palencia, pero si hubiera sido la de Soria, habría empezado a trabajar en un bibliobús. El día que me incorporé a trabajar en la BPE de Palencia, era el cumpleaños de una de las personas que trabajaban en el Centro Coordinador de Bibliotecas, responsable de los bibliobuses de la provincia palentina, que compartía ubicación con la biblioteca. A partir de ahí y durante el tiempo que trabajé en aquella biblioteca, cada día veíamos como salían y volvían de sus rutas las compañeras y compañeros, "bibliobuseras" y conductores, incluso, la única vez que en mi trabajo he visto imprimir juegos de fichas, eran para los catálogos de los bibliobuses que aún no estaban automatizados. Por otra parte, hace mucho también (de todo hace mucho ya), con motivo de los eventos para celebrar la mayoría de edad de mi siguiente destino, la Biblioteca Pública de Salamanca, tuvimos la suerte de contar con Lorenzo Soler, director y productor del breve pero muy emotivo documental "El viaje de los libros", que muestra el trabajo de los

Bajo el principio de que el acceso abierto a los resultados de investigación acelera el avance del conocimiento, todos los contenidos de la edición electrónica de CLIP se distribuyen bajo una licencia de uso y distribución Creative Commons Reconocimiento-NoComercialCompartirlgual 3.0 España (CC BY-NC-SA 3.0 ES). 
compañeros de los bibliobuses en la provincia de Soria (aquel puesto que yo misma pude haber ocupado) y que fervientemente os recomiendo. Hay otros reportajes, todos ellos muy emocionantes, porque este servicio es duro, pero es emocional, es cercano, es vida y quién lo atiende es familia, la gran familia de los bibliobuses (tanto a nivel nacional como internacional), como he tenido la suerte de comprobar y sentir gracias a las ocasiones en que nuestro entrevistado de hoy, Roberto Soto, me ha invitado a participar en alguno de sus eventos.

Este 2021 estaba prevista la celebración del Décimo Congreso Nacional de Bibliotecas Móviles, con el lema “Los Bibliobuses quieren”, pero a falta de esa presencia física, han aplazado también el ordinal para cuando se recupere la misma, y se han permitido la licencia de denominar al del presente año como "Pre-Décimo". Durante los días 16, 23 y 30 de octubre, se han celebrado en libre acceso tres sesiones que han demostrado, una vez más, que los Bibliobuses quieren, pero no sólo eso, sino que quieren SER, quieren SER MÁS y quieren SER BIBLIOTECAS TRANSOCEÁNICAS.

Pero a pesar de ser el $X$ congreso que se celebra con carácter bienal y a pesar de que los bibliobuses no solo quieren ser, sino que SON de hecho, y de que hay una nutrida red en casi todo el territorio nacional, quizá es un servicio un poco desconocido aún entre el conjunto de la ciudadanía e incluso entre los propios colegas de profesión que no nos dedicamos de forma específica.

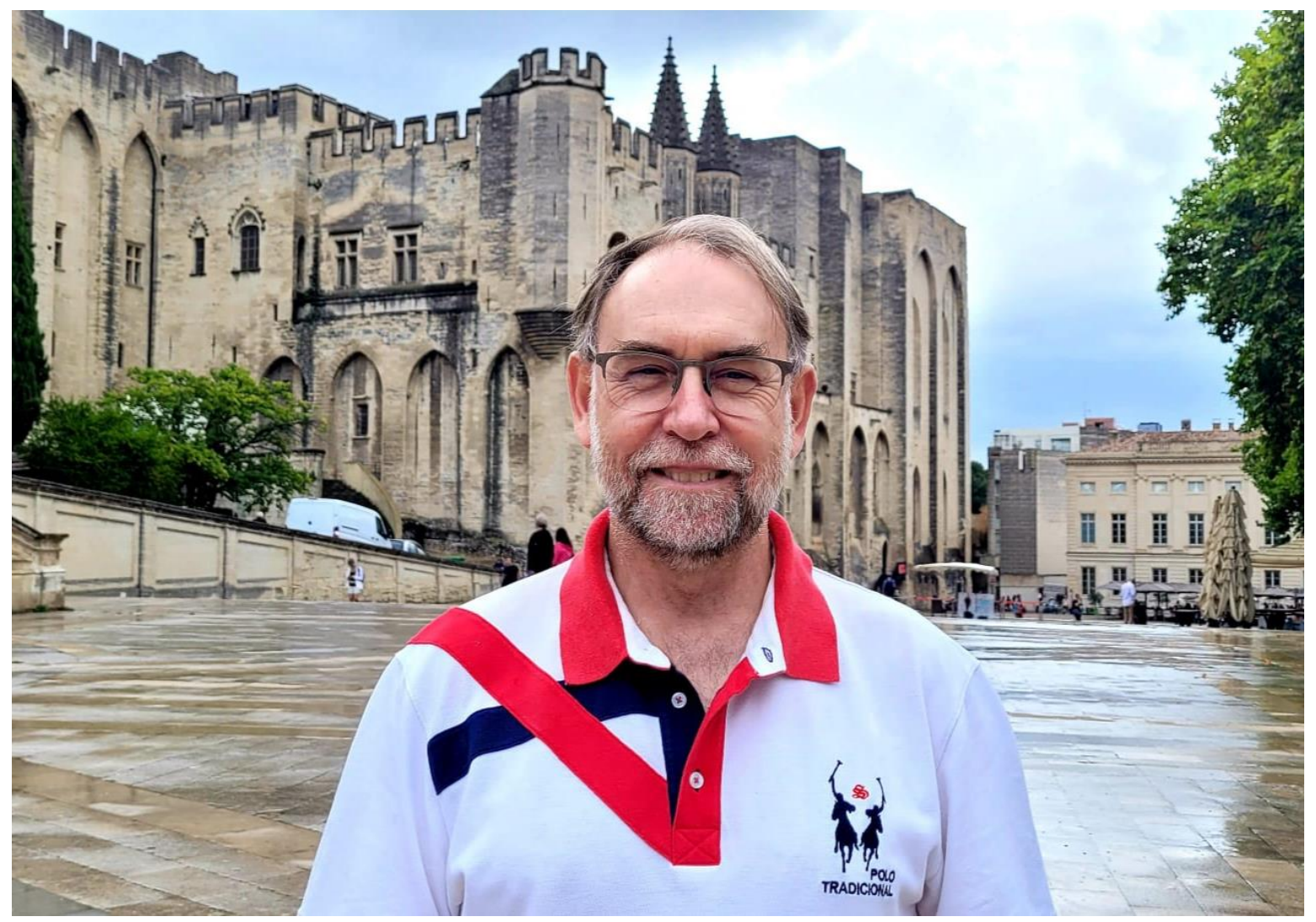

Figura 1: Roberto Soto 
Vamos a empezar con lo obvio, con lo fácil, ¿qué es un bibliobús? Y lo que es más importante, ¿para qué sirve?, ¿cuál es su misión y visión?, ¿qué servicios proporcionan?, ¿quiénes son sus usuarios?

Un bibliobús es una biblioteca que se mueve, que circula por un territorio en busca de sus usuarios, a los que llega para proporcionarles los beneficios de la biblioteca pública, allí donde no existe otra alternativa para ello. En España su forma más común es la de un autobús, de ahí su nombre, pero no es más que una modalidad de biblioteca móvil de las muchas que existen por el mundo, instaladas en furgonetas, barcos, aviones, lanchas, trenes, animales o incluso a la espalda de personas sin más.

Aunque lo más llamativo, y también lo más determinante de su naturaleza es la movilidad, lo cierto es que la verdadera relevancia de un bibliobús se produce cuando está parado, es decir, cuando llega a sus paradas y se convierte en la biblioteca de dicha localidad.

También la movilidad provoca que, para quien no conoce este servicio, pueda pensar de primeras que no hay una planificación, pero más bien es todo lo contrario, las fechas de visita, los tiempos, las localidades, los lugares de parada en las mismas, todo está puntualmente medido y planificado de antemano, generalmente con un año de antelación, y publicitado, para que el usuario tenga toda la información precisa con el fin de conseguir un aprovechamiento lo más óptimo del servicio. $Y$, por supuesto, la pieza clave es el personal, profesionales con los suficientes conocimientos, capacidades y habilidades profesionales, que no serían nada sin motivación a prueba de todo, don de gentes y un espíritu de servicio inquebrantable.

Como en cualquier biblioteca pública, desde el bibliobús se ofrece un servicio de préstamo a domicilio, si bien la mayor parte de las veces como resultado del servicio de prescripción lectora. La cualidad de las personas que acuden al bibliobuses, generalmente ancianas e infantiles, junto a la especial relación de proximidad, de complicidad diría yo, se combinan para que en el bibliobús exista un "préstamo de amigo", o lo que es lo mismo, el consejo bibliotecario en estas lides suele aceptarse con naturalidad y apreciarse en gran medida.

No obstante, los bibliobuses también tienen sus propias programaciones de animación a la lectura, a la escritura y de extensión cultural, por el mismo hecho básico de dirigirse a una parte de la ciudadanía para los que constituyen la única puerta de acceso a la cultura de calidad.

Si la llegada del bibliobús, utilizando la expresión de muchos usuarios, es una fiesta, la organización de nuestras actividades supone un auténtico acontecimiento, en lugares por lo general abandonados del resto de circuitos culturales, y con grandes carencias en el resto de servicio públicos.

Desde este contexto, se hace especialmente necesario llevar desde los típicos cuentacuentos hasta los talleres de educación digital, pasando por los propios de creación del conocimiento (laboratorios ciudadanos, fab lab, ...) en un intento humilde por retener el talento local, y sin olvidar los clubes de lectura, que tanto fortalecen la comunidad.

Aunque también existen bibliobuses urbanos, la mayoría de los bibliobuses españoles tienen un marcado carácter rural y, de hecho, gracias a la calamitosa situación de despoblación, envejecimiento y desertización de nuestro medio rural, en mucho casos se convierte en el cierre de los servicios bibliotecarios públicos en muchas de las zonas que sirven.

Una parte importante de sus usuarios, especialmente en aquellas localidades que conservan la escuela, son infantiles, en estrecha colaboración con el profesorado. De igual modo, los mayores son el otro grupo dominante. También existen otros grupos de edad, lógicamente, 
si bien no tan numerosos, entre los que destacan mujeres maduras, que suelen coincidir con las madres de los primeros y con las cuidadoras de los segundos, de existir el caso.

Lo que nunca olvidamos es que, por imperativo constitucional, el acceso a la cultura es un derecho fundamental en España, y nosotros somos la mejor solución bibliotecaria por su potencia, su calidad y su flexibilidad para conseguirlo. Los poderes públicos promoverán y tutelarán el acceso a la cultura, a la que todos tienen derecho, dice el artículo el artículo 44 de la Constitución, y nosotros somos los poderes públicos con las herramientas precisas para asegurar esa igualdad, conscientes de que la cultura no es un adorno, es un elementos de progreso, de fortalecimiento de las personas y sus comunidades, que contribuye activamente a reducir cualquier brecha y que mejorar sustancialmente la calidad de vida y el futuro de las gentes.

\section{¿Cómo y cuándo empieza tu relación con los Bibliobuses?}

Todo sucedió en 1991, al aprobar las oposiciones de encargado de bibliobús convocadas un año antes por la Diputación de León. Bibliotecario en trabajos en precario hasta esa fecha, era un opositor a todo lo que salía en el ramo, e igual podría haber terminado en alguna biblioteca pública, universitaria o especializada, pero al final fue en los bibliobuses.

Como en cualquier trabajo, los primeros meses fueron de aprendizaje, puesto que hay muchos detalles que te los desvela la experiencia del día a día, hasta que un día te das cuenta de que, aun disfrutando de lo que haces, pocos secretos te depara el trabajo cotidiano, y necesitas ampliar horizontes profesionales en otros puestos u ocupaciones. Así, durante ocho años, paralelamente, dos tardes por semana puse a funcionar y gestioné la Biblioteca Municipal de San Miguel del Arroyo en régimen de voluntariado, pero esa es otra historia, no menos motivadora, por cierto.

\section{¿Qué es ACLEBIM y cómo empieza tu relación con esta asociación que presides?}

La Asociación de Profesionales de Bibliotecas Móviles nace en 1998 con la intención de defender este tipo de servicios y a su personal, con las motivaciones propias de este tipo de organizaciones, tales como el interés por la situación y derechos laborales del gremio, su capacitación profesional y la potenciación de la visibilidad y profesionalización de los bibliobuses.

La tarea fue difícil desde un principio, pues nuestro punto de partida contaba con convencer a una gran parte de la colectividad de bibliotecarios que los bibliobuses también eran bibliotecas y su personal, profesionales del ramo.

Para conseguir estos objetivos, nos propusimos desde el primer momento dotar a nuestro trabajo diario en ruta de la pátina profesional precisa, para lo que, en un primer momento, conseguimos publicar el primer manual de bibliobuses existente en el ámbito hispano (La biblioteca móvil. Trea, 2001), cuyos autores eran todos bibliotecarios de ruta.

A continuación, y en la misma línea, convocamos el Primer Congreso Nacional de Bibliotecas Móviles, donde, por primera vez, conseguimos reunir a la mayor parte del personal que trabajaba en bibliobuses en España (2002). Habíamos iniciado un proceso que hasta ahora ha celebrado diez ediciones, y que ha procurado el crecimiento sistemático de la literatura científica sobre servicios bibliotecarios móviles al tiempo que el nacimiento y fortalecimiento de la Gran Familia de los Bibliobuses. Pasamos de desconocer si existían bibliobuses en la provincia 
de al lado, a entablar una verdadera red de contactos personales y profesionales que dura hasta nuestros días.

El siguiente paso fue la apuesta por la cuantificación de nuestro trabajo desde el mismo punto de vista que lo hacían las demás bibliotecas, pero considerando en ello los elementos que, sin ser bibliotecarios, inciden directamente en el desarrollo de nuestros servicios. Así nació nuestra Propuesta de indicadores de rendimiento para Bibliotecas Móviles (2005)

A partir de ese momento, seguimos trabajando sin descanso en nuestro afán por lograr, entre otras cosas, la puesta en marcha de los Premios ACLEBIM de Bibliotecas Móviles, la traducción al español de las Pautas IFLA sobre Bibliotecas Móviles, el Día del Bibliobús en España, el primer sello postal dedicado al Bibliobús, el Premio Nacional al Fomento de la Lectura... así como una red de contactos internacionales con países como Chile, Argentina, México, Portugal, Croacia o Países Bajos que no hacen más que fortalecer nuestra labor diaria en ruta.

No hemos de olvidar la relevancia de la web de ACLEBIM, no solo por constituir el sitio de consulta obligada sobre los bibliobuses españoles, por su directorio, y sus recursos variados, sino por contener la mayor bibliografía sobre servicios móviles en texto completo en español.

Es cierto que queda mucho por hacer, especialmente en la homogeneidad de la situación profesional de nuestros compañeros, mal de muchos gremios bibliotecarios que no nos consuela, pero que fue unos de los detonantes principales del nacimiento de ACLEBIM.

Hacia 1992 el desconocimiento de esta especialización de la biblioteca pública era absoluto, incluso para lo que trabajábamos en ella, no sólo en cuanto a los efectivos reales existentes en España, sino también en lo relativo a sus condiciones profesionales y de servicio. $Y$ con una referencia de literatura científica escasa y salida de autores que nunca habían trabajado en un bibliobús.

En ese año solicité información al respecto a la Biblioteca de Castilla y León, como cabecera de nuestro sistema regional, para, con esos datos, convocar a todo el personal que quiso personarse a una reunión para conocernos. Ese fue el germen de ACLEBIM, que no nacerías hasta ocho años después, cuando por fin decidimos formalizar aquellos encuentros anuales en los que básicamente aprovechábamos para ponernos al día y "llorar" sobre lo mal que nos iba.

Ante el nacimiento del Grupo de Trabajo del Ministerio de Cultura encargado de redactar los que serían El servicio de bibliobús. Pautas básicas para su funcionamiento, formalizamos nuestra relación y sustituimos los lloros por el trabajo duro.

Como promotor y defensor de la idea me tocó la presidencia del invento y... hasta ahora.

\section{¿Qué ha pasado en los últimos años en los bibliobuses? ¿Como les ha afectado la} pandemia?

Los bibliobuses, en los últimos años, se han visto integrados de facto y con todos los derechos en los sistemas bibliotecarios regionales a los que pertenecen, algo que hace veinte años no estaba muy extendido; así, forman parte de sus sistemas informáticos de gestión, e igualmente, su personal se ha equiparado con sus similares propios de las bibliotecas estables.

Como ya he dicho antes no es un mundo color de rosa, pero consecuciones como las anteriores han venido de la mano de la modernización de las flotas, de una mayor planificación y de una mayor visibilidad, tanto dentro como fuera de la profesión, hasta el punto de contar con una sección específica de bibliobuses en las estadísticas oficiales sobre bibliotecas públicas del Ministerio de Cultura, aspecto este último que antes desarrolló ACLEBIM con sendos estudios sobre la situación de los profesionales en los bibliobuses españoles. 
En el caso concreto de la pandemia, como para todos, ha sido un momento muy difícil para los bibliobuses, especialmente por quedarse privados de uno de sus valores fundamentales, como es el contacto físico, la presencialidad; pero, además, el hecho de ocuparnos habitualmente de los más vulnerables ha sido especialmente duro no poder acudir cuando más nos han necesitado.

Ha habido servicios que activaron o intensificaron, según los casos, sus prestaciones digitales, y también analógicos de comunicación, como la presencia en redes, mensajes de whatsapp, llamadas telefónicas o el préstamo por correo. También otros se echaron a las carreteras en cuanto la normativa sanitaria les autorizó, llevando a sus usuarios una sensación de normalidad todavía inexistente, pero de gran valor, para los que los esperaban.

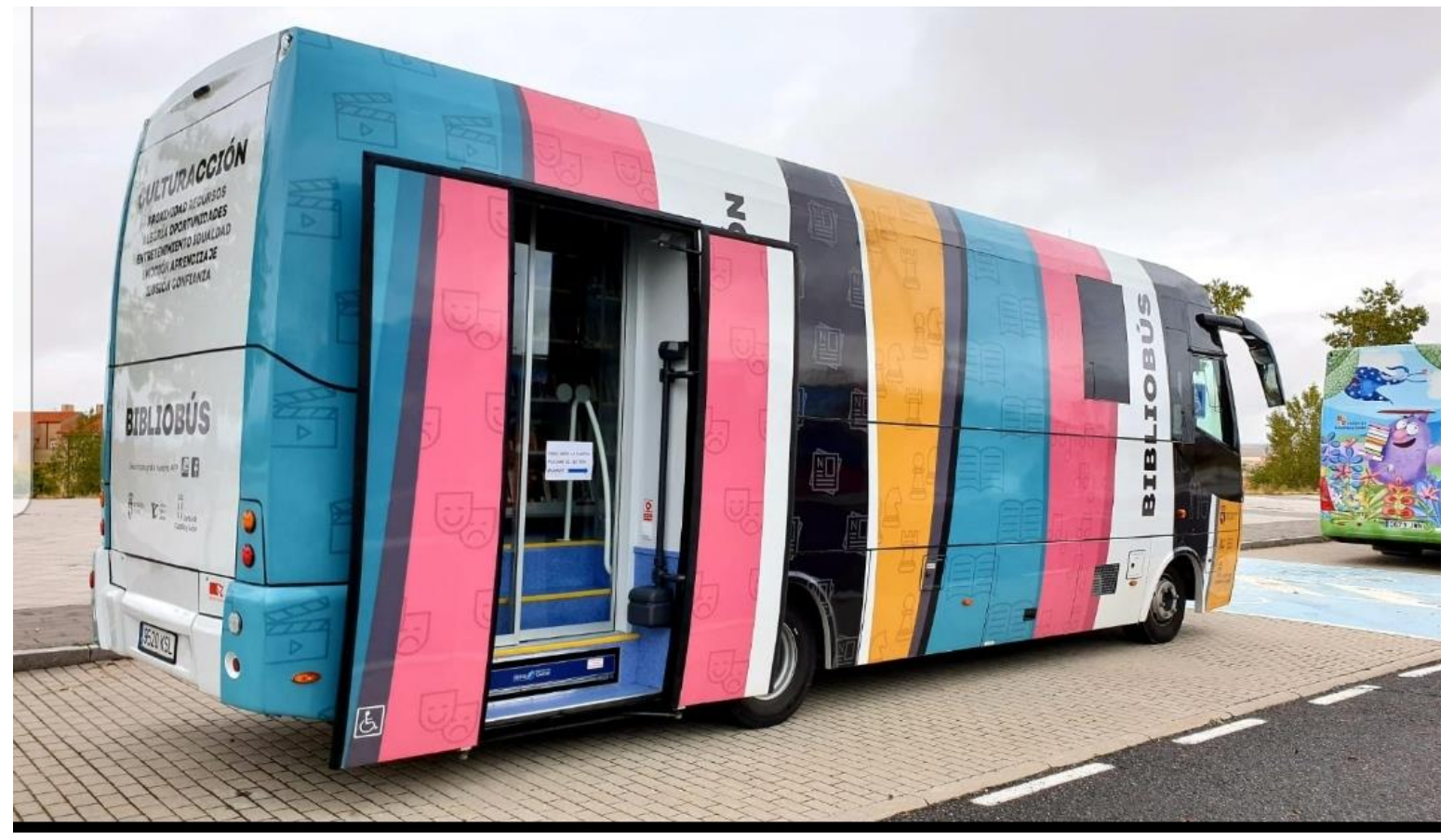

Figura 2: Bibliobuses durante el $9^{\circ}$ Congreso Nacional de Bibliotecas Móviles celebrado en la Biblioteca Pública de Segovia en 2019

\section{¿Qué papel tienen los bibliobuses en la España rural, vacía o, mejor dicho, vaciada?}

El papel de los bibliobuses en la España vacía es fundamental, solo con pensar que su servicio está definido para los desheredados, los que no figuran habitualmente en el catálogo de los servicios público, que llegan donde nadie llega, que son el único acceso directo a la cultura de calidad para la población a la que sirven, solo con todo ello, los bibliobuses son instrumentos esenciales para el desarrollo de la comunidad allí donde paran y absolutamente necesarios donde no existen.

La cultura es una parte de la solución al problema demográfico. La despoblación, la desertización y el envejecimiento del interior de nuestro país son fruto de políticas en favor de la atracción urbana sin sostenibilidad alguna, que han privado al medio rural de casi todos los servicios que hacen posible una vida completa y cómoda para sus habitantes. 
Los jóvenes huyen en busca de trabajo y oportunidades, y de una igualdad que el sistema les priva si se quedan en el campo. El sistema educativo ha acabado preparándoles para el éxodo desde sus primeros años, con un transporte escolar inhumano desde edades muy tempranas para su distribución entre los centros rurales agrupados (CRA), en primera instancia, para después pasar a las cabeceras de comarca en la secundaria, y terminar en las capitales de provincia para recibir estudios universitarios.

Un pueblo sin niños es un pueblo triste... y resignado a su aciago futuro. El bibliobús es el agente cultural más activo, cercano y sostenible para frenar esta tendencia y fomentar el desarrollo, el despertar de las comunidades de, cada vez, menos vecinos. O quizá sea el bibliobús un agente social que se sirva de la cultura para lograr sus objetivos...

El caso es que la presencia de los bibliobuses en las distintas localidades siempre es una fiesta, es un revulsivo, una estupenda excusa para relacionarse, para la convivencia activa de sus vecinos, para, en suma, el fortalecimiento de la comunidad misma.

Pero los bibliobuses pueden y van más allá, ya que no sólo propician, por ejemplo, la capacitación digital, sino la conexión misma, la consulta libre y gratuita de internet, un amplio acerbo documental, la orientación experta de su personal, una vasta programación de actos culturales y de talleres formativos.

Hay bibliobuses especializados en el trato con los mayores, otros en el servicio a los colegios, los hay que fortalecen la identidad de la comunidad recogiendo, procesando, conservando y difundiendo su patrimonio inmaterial; sin dejar atrás las relaciones propias del grupo con los clubes de lectura y otras celebraciones.

Los bibliobuses son un soplo de aire fresco a la España vacía, un impulso de su autoestima, de que no todo está perdido, y la posibilidad de recuperar, al menos, parte de lo abandonado.

La sostenibilidad de los bibliobuses es incuestionable, baste considerar que de cada cuatro habitantes atendidos por una biblioteca pública en España, uno pertenece a un bibliobús. Son once millones trecientas mil personas para los que es su único cordón umbilical con la modernidad y la igualdad de derecho y oportunidades.

Son muchos los servicios que precisamos para combatir la despoblación, pero sin cultura, y sin su arma más versátil y poderosa, toda medida al respecto carecerá de alma y, por tanto, tendrá una corta vigencia.

¿Qué opinas de los servicios quizá menos bibliotecarios, pero muy útiles y de servicio público, como los que prestan los compañeros del vecino Portugal, por ejemplo? ¿Esos servicios que van mucho más allá de los servicios tradicionales de préstamo a domicilio y consulta documental, permitiendo a sus usuarios acceder a Internet, imprimir documentos, pero también pagar servicios como agua, luz, etc., a través de un cajero automático, cargar tu teléfono móvil y, en ocasiones te acompaña un técnico de la Unidad Móvil de Salud para realizar los controles?

Me parece una evolución natural de nuestros servicios, teniendo siempre presente el contexto de precariedad en el que desarrollan su labor. Si desde el bibliobús se está preparado para la formación a lo largo de la vida, si ofrecemos capacitación digital, si incluso alfabetizamos a la gente, lo normal es que pongamos en sus manos libros para leer, ordenadores y otros dispositivos para manejar, y todo aquello que les ayude a mejorar su bienestar. 
Por otra parte, el aprovechamiento de nuestra logística para la dispensa de otros servicios no estrictamente bibliotecarios constituye un acto de racionalización de la administración pública, de optimización de recursos y de pura sostenibilidad y de cooperación simbiótica con otros sectores.

La mayor parte de los bibliobuses tienen una titularidad pública (en España, todos), y la Administración ha de modernizarse en su esfuerzo continuo por adaptarse a la sociedad a la que sirve, una sociedad cada día más cambiante y poliédrica. Los servicios públicos no pueden seguir siendo compartimentos estancos, especializados en un tema muy concreto, y dejar en manos de otros el resto de los factores cuyo conjunto constituye la vida diaria de la ciudadanía.

La oferta de estos nuevos servicios desde los bibliobuses es una oportunidad maravillosa de ampliar el volumen de usuarios, y de transmitir el valor real de nuestras actividades, de demostrar de qué somos capaces y hasta qué punto se puede contar con nosotros.

Si hay bibliotecas que crean conocimiento, donde se puede tomar un café, donde se puede sacar en préstamo semillas u obras de arte, e incluso paraguas cuando llueve, ¿por qué un bibliobús no puede ofrecer a sus usuarios aquello que más necesitan para que su vida sea más fácil?

\section{¿Cómo ves, entonces, que los Bibliobuses de Salamanca vayan a incluir cajeros automáticos entre sus servicios habituales?}

Está dentro de la misma línea que estamos comentando, pero con una diferencia fundamental, como es el hecho de que su instalación no ha obedecido a criterios de servicios bibliotecario, ni se ha diseñado para su conjunción en el mismo espacio, como prueba que el cajero se ha dispuesto para su consulta en la calle, sin necesidad de entrar en el bibliobús.

La instalación de estos cajeros ha supuesto para los Bibliobuses de Salamanca la solución a problemas endémicos que nadie hacía por resolver en la entidad política de la que dependen que, sin embargo, con el fin de garantizar el servicio bancario se han arreglado de un plumazo, se ha mejorado el sueldo del personal, se ha implementado personal de reemplazo y hasta se inaugurará un nuevo bibliobús a mayores.

Bienvenido sea todo, pero tristemente no corresponde a inquietudes de servicio como las que venimos explicando en esta entrevista, sino a la intención de que el servicio bancario, que, por ley ni siquiera es gratuito para la población, funcione siempre y en todo lugar.

Espero que todo el proyecto funcione muy bien, puesto que, de no ser así, qué acabarían pensando sus promotores del papel real de sus bibliobuses.

\section{¿Cómo ves el futuro de los bibliobuses? Así, en general...}

Con las experiencias de nuestro país y con las que vemos en el extranjero, no tengo más que una visión absolutamente esperanzadora sobre el futuro de los bibliobuses.

Durante mucho tiempo se los denostó por sus limitaciones de espacio y de tiempo. Ambas han quedado bien resueltas con las nuevas tecnologías y comunicación por internet (webs interactivas y redes sociales). Poco tenemos que añadir sobre su rentabilidad social y su sostenibilidad. Sin embargo, la mayor limitación es la procedente de los prejuicios que todavía existen para este tipo de servicios bibliotecarios. Podemos concebir una UVI móvil pero no una biblioteca móvil... 
La realidad demográfica de nuestros pueblos, las tecnologías de la comunicación y almacenamiento de información, las capacidades técnicas de los nuevos vehículos, la capacidad de adaptación a las diferentes realidades, el aumento de su visibilidad, la empatía que provocan en la población, su experiencia continuada, su abundante y actualizada literatura científica y su demostrada pertinencia y operatividad como servicios todoterreno hacen de los bibliobuses una bibliotecas con un futuro halagüeño.

Por todo ello no tengo ninguna duda sobre la longevidad de los bibliobuses. Actualmente contamos con un problema fundamental en el envejecimiento de su personal para el panorama español. Es una mal extendido en toda la Administración Pública que habrá de hacer frente en breve, pero que en el caso de los bibliobuses supondrá una renovación de plantillas que oxigenará los servicios, con una visión y forma de trabajar que quizá no estemos alcanzando los que ya somos veteranos.

\section{¿Puedes contarnos alguna anécdota que hayas vivido?}

Anécdotas hay muchas, pero todas coinciden en un elemento muy valorado por todos nosotros como son las demostraciones de cariño que el público tiene al servicio de bibliobuses, y como muestra valgan dos ejemplos:

Recuerdo cómo una vez, en plena alta montaña, la nieve no nos permitió dar la vuelta y no tuvimos más remedio que tirar hacia adelante hasta llegar al primer pueblo que teníamos pendiente en visitar, allí nuestros usuarios nos abroncaron por la temeridad y con sus palas retiraron la nieve que nos estorbaba para maniobrar y salir de allí cuanto antes para poder regresar a casa.

Otras veces hemos asistido a la triste situación de conocer el fallecimiento de algunos de nuestros usuarios Por boca de sus familiares, cuando acudían a devolvernos los libros por instrucciones expresas del finado.

Detalles como estos te revelan hasta qué punto es importante en la vida de las personas el trabajo que realizas desde el bibliobús.

\section{Sobre la autora}

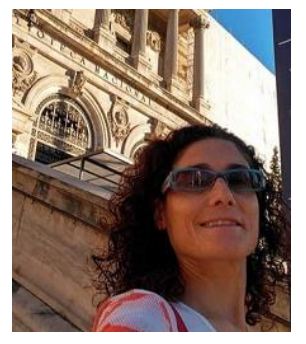

FELICIDAD CAMPAL

Ayudante de bibliotecas en la Biblioteca Pública de Salamanca Bibliotecaria, profesora, cursillista, tallerista, congresista y comunicadora en constante aprendizaje y renovación permanente. Convencida y confesa del poder formativo, democrático social, integrador e igualador de las bibliotecas, si trabajamos todas juntas. 